\title{
A Finitely Generated Module Over a Discrete Valuation Domain
}

\author{
Dwi Mifta Mahanani \\ Department of Mathematics Brawijaya University \\ Email: mdwimifta@yahoo.com
}

\begin{abstract}
This article discusses some properties of a finitely generated module over a PID and that over a valuation domain which are equivalent. In general, PID is not a valuation domain and also a valuation domain is not a PID. But there is a domain which is PID as well as a valuation domain. The domain is called a discrete valuation domain (DVR). Therefore, the equivalent properties which will be considered are obtained from a finitely generated module over a DVR. Those properties are related to a decomposition into direct sum of cyclic submodules and height of an element of the module.
\end{abstract}

Keywords: DVR; finitely generated module; height of element; PID; valuation domain

\section{INTRODUCTION}

In general, the properties of an $R-$ module depend on the properties of ring $R$. For example, every finitely generated module over PID can be decomposed into a direct sum of cyclic submodules. However, that property does not satisfy in every finitely generated modules over a valuation domains. That is to say, not all of those modules can be decomposed into a direct sum of cyclic submodules. Moreover, those which can be decomposed are those which the Goldie dimension is equal to the length of their series of $R D$-submodules. The example of finitely generated modules over a valuation domain which can not be decomposed can be seen in [5].

In general, a PID is not a valuation domain. An integral domain $\mathbb{Z}$, for instance, is a PID but not a valuation domain because there are two ideals of $\mathbb{Z}, \mathbb{Z} 2$ and $\mathbb{Z} 5$, which are not subset each other. Otherwise, a valuation domain is not a PID. Note the example below.

\section{Example}

Let $K$ be a field with transcendental $x, K_{i}=K\left[x^{\frac{1}{2^{i}}}\right], P_{i}=K\left[x^{\frac{1}{2^{i}}}\right] x^{\frac{1}{2^{i}}}$ where $i=0,1,2, \cdots$. It can be seen obviously that $K_{i}$ is a PID and $P_{i}$ is it's prime ideal. Moreover, there are domains

$$
\begin{aligned}
O_{i} & =K_{i_{P_{i}}} \\
& =\left\{\frac{f\left(x^{\frac{1}{2^{i}}}\right)}{g\left(x^{\frac{1}{2^{i}}}\right)} \mid f\left(x^{\frac{1}{2^{i}}}\right) \in K_{i}, g\left(x^{\frac{1}{2^{i}}}\right) \in K_{i}-P_{i}\right\} .
\end{aligned}
$$


A domain $O=\mathrm{U}_{i} O_{i}$ is a non-Noetherian valuation domain. Hence $O$ is not a PID. The detail of the example can be seen in [3] and [6].

Meanwhile, there is a domain which is a PID as well as a valuation domain. In [2], this domain is called discrete valuation domain (DVR). Because a DVR is an intersection of two different domains, then there must be some properties of finitely generated modules over a PID and those over a valuation domain which are equivalent. These equivalent properties will be discussed in the result section.

\section{MATERIALS AND METHODS}

The method which is used to write this article is by literature studying on some papers and books. There are some properties of a module over a DVR which have been discussed in the papers and the books. It will be developed some new properties by considering the previous properties. Before we discuss the results, we have to know some definitions and properties that we use in the proof of the results. The readers are assumed to know about the definition of a module over a ring and some basic properties of a module. Therefore, in this article there are no definition and basic properties of a module over a ring.

Definition 1 [1, pp. 115]

Let $M$ be a finitely generated $R$-module. The minimal number of generators of $M$ is denoted by $\mu(M)$. If $M$ is not finitely generated as $R$-module, then $\mu(M)=\infty$.

Unlike in vector spaces, in modules, not all of those have a basis. For those which have a basis are called free modules as which have been explained in [8]. We follow [1] that every basis of a free module over a PID contains $\mu(M)$ elements .

There is a submodule of modules over a valuation domain which has an important role in those modules. This kind of submodule is an $R D$-submodule. The definition of this submodule is presented below.

Definition 2 [4, pp. 38]

Let $N$ be an $R$-submodule of a module $M$. This submodule is a relatively divisible submodule ( $R D$-submodule) of $M$ if $r N=N \cap r M$ for all $r \in R$.

There are some properties of $R D$-submodule which will be used to prove the equivalence of two theorems in a finitely generated free module over a PID and in that over a valuation domain. These properties will be given without any proof.

Lemma 3 [4, pp. 39]

Let $M$ be an $R$-module and $L, N$ are $R$ - submodules of $M$. Then these properties are satisfied.

1 Direct summand of $M$ is an $R D$-submodule of $M$.

2 If $L \leq N \leq M$ and $L, N$ are $R D$-submodules of $M$, then $L$ is an $R D$-submodule of $N$.

Using those properties of $R D$-submodule to proof that a submodule is an $R D$-submodule is easier than using the definition. The examples related to 
$R D$-submodule can be seen below.

1. Ring $\mathbb{Z}_{15}$ can be assumed to be a $\mathbb{Z}$-module. Note that $\mathbb{Z}_{15}=\mathbb{Z} \overline{3} \oplus \mathbb{Z} \overline{5}$. Then by lemma $3, \mathbb{Z} \overline{3}$ and $\mathbb{Z} \overline{5}$ are $R D$-submodules of $\mathbb{Z}_{15}$.

2. A domain $\mathbb{Z}$ can be considered as a module over itself. We know that every submodule of this module is an ideal of $\mathbb{Z}$. Furthermore, every ideal of $\mathbb{Z}$ is generated by single element (principal ideal). Therefore every two nonzero ideals of $\mathbb{Z}$ always have a nonzero intersection element. Hence, $\mathbb{Z}$ as a $\mathbb{Z}$-module does not have any proper nonzero $R D$-submodule.

In a DVR, there is a unique element (up to associates) which has an important role to define a height of an element of a module over this domain.

Definition 4 [1, pp.98]

Let $R$ be a ring and $r \in R$. An element $r$ is a non nilpotent element of $R$ if there is no positive integer $n$ such that $r^{n}=0$.

Because of the uniqueness of a non nilpotent in a DVR, we can write every element in this domain by a multiplication of a unit element and this non nilpotent element. Moreover, this can also be written uniquely. Note the lemma below.

Lemma 5 [7, pp. 5009]

Let $a$ be a nonzero element of a DVR $R$. Then $a$ can be written uniquely as $a=u p^{k}$ where $u$ is a unit of $R, p$ is a non nilpotent element of $R$ and $k \in \mathbb{Z}_{\geq 0}$.

The theorem below explains about the decomposition of a finitely generated module over a PID into a direct sum of finite cyclic submodules. Moreover, the annihilator of generators of these cyclic submodules can be arranged such that they form a non decreasing chain. Note this theorem below.

Theorem 6 [1, pp. 156]

Let $M \neq 0$ be a finitely generated module over a PID $R$. If $\mu(M)=n$, then $M$ is isomorphic to

$$
R w_{1} \oplus R w_{2} \oplus \cdots \oplus R w_{n}
$$

such that

$$
\operatorname{Ann}(M)=\operatorname{Ann}\left(w_{n}\right) \subseteq \cdots \subseteq \operatorname{Ann}\left(w_{2}\right) \subseteq \operatorname{Ann}\left(w_{1}\right) \neq R .
$$

Moreover, for $1 \leq i \leq n$,

$$
\operatorname{Ann}\left(w_{i}\right)=\operatorname{Ann}\left(\frac{M}{R w_{i+1}+\cdots+R w_{n}}\right)
$$

Different from a finitely generated module over a PID, in that over a valuation domain, we can find a series of $R D$-submodules. Moreover, the chain can be chosen so 
that the annihilators of the factors forms a non decreasing chain.

Theorem 7 [5, pp. 1798]

Let $M \neq 0$ is a finitely generated module over a valuation domain $R$. There exist a series of $R D$-submodule of $M$

$$
0=M_{0}<M_{1}<M_{2}<\cdots<M_{n}=M
$$

such that

1. every $M_{i}$ is an $R D$-submodule of $M$,

2. every factor module $M_{i+1} / M_{i}$ is cyclic.

Moreover, it can be chosen that

$$
\operatorname{Ann}(M)=\operatorname{Ann}\left(M_{1}\right) \leq \operatorname{Ann}\left(\frac{M_{2}}{M_{1}}\right) \leq \cdots \leq \operatorname{Ann}\left(\frac{M_{n}}{M_{n-1}}\right)
$$

There is a possibility for a finitely generated module over a valuation domain to have more than one $R D$-submodules series. Nevertheless, there is another property of this module stating that every two $R D$-submodules series are isomorphic. Hence, we can choose any series of $R D$-submodule.

Now consider a finitely generated free module over a PID. In this module, an $R D$-submodule is the same as a complemented submodule. Note the theorem below.

Theorem 8 [1, pp. 172]

A submodule of a finitely generated free module over a PID is an $R D$-submodule if and only if it is complemented.

Theorem 8 will be used to construct a direct sum of cyclic submodules of a finitely generated free module over a DVR.

Theorem 9 [5, pp. 1801]

Let $M$ be a finitely generated $R-$ module where $R$ is a valuation domain. Let $0 \neq x \in M$ be any element of $M$. There exist $r \in R$ such that $x \in r M-r P M$ where $P$ is a maximal ideal of $R$.

The theorem above ensures the existence of an element $r \in R$ which has a property that $x$ always in $r M$ but not in $r P M$. If there is another element $s \in R$ which satisfies the property in the theorem 9, then the ideal $R r$ will be equal to ideal $R s$. Theorem 9 and that argument motivate a definition of height of an element in a module over a valuation domain. The definition is presented below.

Definition 10 [5, pp. 1802]

Let $M$ be a finitely generated $R-$ module where $R$ is a valuation domain. Let $0 \neq x \in M$ be any element of $M$. Height of $x$, which is denoted by $h_{M}(x)$, is defined as an ideal $R r$ such that $x \in(r M-r P M)$ where $P$ is a maximal ideal of $R$. Height of 0 is defined as a zero ideal.

The definition of height of an element of a finitely generated module over a 
valuation domain and that over a DVR are different. In a module over a valuation domain, the height of an element is in the form of an ideal. However, in a module over a DVR, it is a non negative integer. In fact, DVR is a special case of valuation domain. Those two statements actually are equivalent. This equivalence will be proven in the next theorem. But before doing the proof, note the definition of height of an element of a module over a DVR.

Definition 11 [7, pp. 5033]

Let $a \in M$ be any nonzero element where $M$ is an $R$-module over a DVR. Height of $a$ is the greatest non negative integer $n$ which equation $p^{n} x=a$ is soluble. If there is no such integer, it means the height is $\infty$. Element $p$ in the equation is a non nilpotent element in $R$.

From the definition above, the height of zero element in $M$ is $\infty$. It is because there is no greatest non negative integer $n$ such that $p^{n} x=0$ is soluble.

\section{RESULTS AND DISCUSSION}

We know that a DVR is a Noetherian valuation domain. Therefore, every property in a module over a valuation domain always satisfies in that over a DVR. One of those properties is the existence of a series of RD-submodules. Note an illustration below.

Let $M$ be a finitely generated $R$-module over a PID and $\mu(M)=n$. It will be constructed a series of $R D$-submodule of $M$. Based on theorem 6 , module $M$ is isomorphic to

$$
R w_{1} \oplus R w_{2} \oplus \cdots \oplus R w_{n}
$$

With this direct sum, we can make a series. Observe this series below.

$$
0=M_{0}<R w_{n}<R w_{n} \oplus R w_{n-1}<\cdots<R w_{n} \oplus \cdots \oplus R w_{2} \oplus R w_{1}=M
$$

Based on lemma 3, submodule $R w_{n} \oplus R w_{n-1} \oplus \cdots \oplus R w_{k}$ where $1 \leq k \leq n$ is an $R D$-submodule of $M$. Hence, there exist a series of $R D$-submodule of $M$.

Observe that

$$
\left(\frac{R w_{n} \oplus R w_{n-1} \oplus \cdots \oplus R w_{k-1}}{R w_{n} \oplus R w_{n-1} \oplus \cdots \oplus R w_{k}}\right)
$$

is cyclic with the generator $R\left(w_{k-1}+\left(R w_{n} \oplus R w_{n-1} \oplus \cdots \oplus R w_{k}\right)\right)$ for $1 \leq l \leq n-1$. Let

$$
r \in A n n\left(\frac{R w_{n} \oplus R w_{n-1} \oplus \cdots \oplus R w_{k-1}}{R w_{n} \oplus R w_{n-1} \oplus \cdots \oplus R w_{k}}\right)
$$

Then $r w_{k-1} \in\left(R w_{n} \oplus R w_{n-1} \oplus \cdots \oplus R w_{k}\right)$. Therefore,

$$
r w_{k-1} \in R w_{k-1} \cap\left(R w_{n} \oplus R w_{n-1} \oplus \cdots \oplus R w_{k}\right)
$$


Because $R w_{k-1}+R w_{k}+\cdots+R w_{n}$ is a direct sum, then it causes $r w_{k-1}=0$. It means that $r \in \operatorname{Ann}\left(w_{k-1}\right)$. Based on theorem 6, Ann $\left(w_{k-1}\right)=\operatorname{Ann}\left(\frac{M}{R w_{k}+\cdots+R w_{n}}\right)$. It is obtained that $r w_{k-2}=r_{k} w_{k}+\cdots+r_{n} w_{n}$ for some $r_{i} \in R$. Note that

$$
\begin{aligned}
r w_{k-2}+\left(R w_{n}+\cdots+R w_{k-1}\right) & =\left(r_{k} w_{k}+\cdots+r_{n} w_{n}\right)+\left(R w_{n}+\cdots+R w_{k-1}\right) \\
& =R w_{n}+\cdots+R w_{k-1}
\end{aligned}
$$

Hence

$$
r \in A n n\left(\frac{R w_{n} \oplus R w_{n-1} \oplus \cdots \oplus R w_{k-2}}{R w_{n} \oplus R w_{n-1} \oplus \cdots \oplus R w_{k-1}}\right) .
$$

Therefore, it is proved that

$$
\begin{aligned}
& A n n\left(\frac{R w_{n} \oplus R w_{n-1} \oplus \cdots \oplus R w_{k-1}}{R w_{n} \oplus R w_{n-1} \oplus \cdots \oplus R w_{k}}\right) \\
& \leq A n n\left(\frac{R w_{n} \oplus R w_{n-1} \oplus \cdots \oplus R w_{k-2}}{R w_{n} \oplus R w_{n-1} \oplus \cdots \oplus R w_{k-1}}\right) .
\end{aligned}
$$

Now, let $0 \neq M$ is a finitely generated free module over a DVR $R$. Based on theorem 7, there exist a series of $R D$-submodule

$$
0=M_{0}<M_{1}<M_{2}<\cdots<M_{n}=M
$$

with every factor module is cyclic. Note that $M_{i}$ is an $R D-$ submodule of $M_{i+1}$ because $M_{i}$ is an $R D$-submodule of $M$ for all $i$. Based on theorem $8 M_{i}$ is complemented in $M_{i+1}$. Therefore it can be written

$$
M_{i+1}=M_{i} \oplus N_{i+1}
$$

Note that $M_{i+1} / M_{i} \cong N_{i+1}$ and $M_{i+1} / M_{i}$ is cyclic for every $i$. Hence $N_{i+1}$ is cyclic. It can be written $N_{i+1}=R a_{i+1}$. Therefore, it is obtained

$$
\begin{aligned}
M & =M_{n-1} \oplus R a_{n} \\
& =M_{n-2} \oplus R a_{n-1} \oplus R a_{n} \\
& \vdots \\
& =R a_{1} \oplus R a_{2} \oplus \cdots \oplus R a_{n}
\end{aligned}
$$

Now consider that $\operatorname{Ann}\left(\frac{M_{i+1}}{M_{i}}\right)=\operatorname{Ann}\left(N_{i+1}\right)=\operatorname{Ann}\left(a_{i+1}\right)$. If we choose the series of $R D$-submodule so that

$$
\operatorname{Ann}(M)=\operatorname{Ann}\left(M_{1}\right) \leq \operatorname{Ann}\left(\frac{M_{2}}{M_{1}}\right) \leq \cdots \leq \operatorname{Ann}\left(\frac{M_{n}}{M_{n-1}}\right)
$$


then we have a chain

$$
\operatorname{Ann}(M)=\operatorname{Ann}\left(a_{1}\right) \leq \operatorname{Ann}\left(a_{2}\right) \leq \cdots \leq \operatorname{Ann}\left(a_{n}\right)
$$

By renumbering, that is $a_{n-i}=w_{i+1}$, we have a chain

$$
\operatorname{Ann}(M)=\operatorname{Ann}\left(w_{n}\right) \leq \operatorname{Ann}\left(w_{n-1}\right) \leq \cdots \leq \operatorname{Ann}\left(w_{1}\right)
$$

Illustration above shows that Theorem 6 and Theorem 7 are equivalent if $M$ is a finitely generated free module over a DVR.

\section{Theorem 12}

In a finitely generated free module over a DVR, theorem 6 and theorem 7 are equivalent. Proof.

Beside the equivalent statement, there are also some properties of the module which related to the property of the domain. In this case, we will assume the domain is a valuation domain. As we know, the properties will also satisfy if the domain is a DVR. The comparable property of ideals of a valuation domain is inherited to submodules of a cyclic module over a valuation domain. Note the lemma below.

\section{Lemma 13}

Let $0 \neq R m$ is a cyclic module over a valuation domain $R$. If $N$ and $N^{\prime}$ are submodules of $R m$, then $N \subseteq N^{\prime}$ or $N^{\prime} \subseteq N$.

Proof.

Let $I=\{a \in R \mid a m \in N\}$ and $J=\left\{b \in R \mid b m \in N^{\prime}\right\}$. These two sets are ideals of $R$. We know that $R$ is a valuation domain. Hence we have $I \subseteq J$ or $J \subseteq I$. Let $x=$ am $\in N$ be any element. Then $a \in I$. If $I \subseteq J$, then $a m \in N^{\prime}$. Therefore, $N \subseteq N^{\prime}$. We can prove analogously for $N^{\prime} \subseteq N$.

By lemma 13, a cyclic module over a valuation domain can not be decomposed into a direct sum of its nonzero submodules. Hence a cyclic module over a valuation domain is an indecomposable module. From this fact, we have this corollary below.

\section{Corollary 14}

If $M$ is a finitely generated module over a DVR $R$, then $M$ is isomorphic to direct sum of cyclic indecomposable submodules.

Proof.

Use theorem 6 and lemma 13. 


\section{Lemma 15}

Non nilpotent element of a DVR $R$ is a prime element.

Proof.

Let $p$ be a non nilpotent element of $R$ and $p \mid(a b)$ where $a, b \in R$. To show that $p$ is a prime element of $R$, we need to show that $p \mid a$ or $p \mid b$. Based on lemma 5, let $a=u_{1} p^{n_{1}}$ and $b=u_{2} p^{n_{2}}$ with $u_{1}, u_{2}$ are unit elements of $R$ and $n_{1} \in \mathbb{Z}_{>0}$ or $n_{2} \in \mathbb{Z}_{>0}$. Then it is obvious that $p \mid u_{1} p^{n_{1}}$ or $p \mid u_{2} p^{n_{2}}$. Hence $p$ is a prime element of $R$.

If there is another prime element of $R$, namely $q$, then every element of $R$ can also be written in the form of $u q^{n}$ uniquely where $u$ is a unit of $R$ and $n \in \mathbb{Z}_{\geq 0}$. Note this explanation below.

If $q$ is another prime element of $R$, then $R q$ is a maximal ideal of $R$. The uniqueness of maximal ideal of $R$ makes $R p=R q$. Hence we have $p=r q$ for some $r \in R$. It means $p \mid r q$. Because $p$ is prime element, then $p \mid r$ or $p \mid q$.

If $p \mid r$, then $p s=r$ for some $s \in R$. We have $p=p s q$. It means $s q=1$. Therefore $q$ is a unit element of $R$. It is contradiction because $q$ is prime element. Therefore, we have $p \mid q$. Let $p t=q$ for some $t \in R$. Then we have $p=r t p$. It means $r t=1$. Hence, $r$ is a unit of $R$. Therefore, $p$ and $q$ are associate.

Definition 10 is defined in a finitely generated module over a valuation domain. It means the definition satisfies also for that over a DVR. Hence the definition 10 and definition 11 must be equivalent for a DVR. But before proving this equivalence, we need the property below.

\section{Lemma 16}

Let $R$ be a DVR and $a$ is a non nilpotent element of $R$. Then $R a$ is a maximal ideal of $R$. Proof.

By lemma 15, $a$ is a prime element of $R$. Then $R a$ is a prime ideal which is a maximal ideal of $R$.

\section{Theorem 17}

Definition 10 and definition 11 are equivalent in a finitely generated module over a DVR. Proof.

Let $P$ be a maximal ideal of $R$ and $a$ be any nonzero element of $M$. Because $R$ is a DVR, then $P=R p$ for $p$ is a non nilpotent element of $R$. Based on definition $10 a \in r M-$ $r P M$ for some $r \in R$. We can write $a=r m=u p^{n} m$ with $u$ is a unit of $R, n \in \mathbb{Z}_{\geq 0}, m \in M$ and $r=u p^{n}$. Because $a \notin r P M=r p M$, then $a \notin p^{n+1} M$. Hence $n$ is the greatest non negative integer such that $p^{n} x=a$ have a solution.

Now let $n$ be the greatest non negative integer such that $p^{n} x=a$ is soluble. It means that $a \in p^{n} M-p^{n+1} M$. Hence based on definition 10 , the height of $a$ is $R p^{n}$. 


\section{CONCLUSIONS}

In a finitely generated free module over a DVR, we can make a series of it's $R D$-submodules by arranging it's cyclic submodules which are the summand of it's decomposition. Furthermore, from this series, we also get a special series which has a property that it's sequence of the annihilator of it's factor is ordered non-decreasingly. Otherwise, if we have a series of $R D$-submodule, we can decompose the module into a direct sum of cyclic submodules. Moreover, these cyclic submodules are indecomposable submodules. Besides, the height of an element of a finitely generated module over a DVR can be considered as an ideal of it's domain or a non negative integer which is the prime power of a generator of the ideal.

\section{REFERENCES}

[1] Adkins, A.A. and Weintraub, S.H., "Algebra An Approach via Module Theory", Springer-Verlag,1992.

[2] Dummit, D.S. and Foote, R.M., "Abstract Algebra", USA: John Wiley \& Sons Inc, 2004.

[3] Knaf, H. (https://math.stackexchange.com/users/2479/hagen-knaf), "Examples of Non-Noetherian Valuation Rings", URL (version: 2014-09-02): https://math.stackexchange.com/q/363201.

[4] Laszlo, F. and Luigi, S, "Modules over Non-Noetherian Domains", Rhode Island: American Mathematical Society, 2001.

[5] Luigi, S. and Paolo, Z., "Finitely Generated Modules over Valuation Ring". Comm. Algebra. 12:15, pp. 1795-1812. 1984.

[6] Mahanani, D.M., "Thesis: Karakterisasi Dekomposisi Modul yang Dibangun secara Hingga atas Daerah Valuasi", ITB, 2014.

[7] Piotr K., Askar A.T., "Modules over Discrete Valuation Domains I", Journal of Mathematics Science, Volume 145, No.4, pp. 5008-5048, 2007.

[8] Roman, S., "Advanced Linear Algebra Third Edition", Springer, 2000. 